the field. Together, these studies provide new information on whole body metabolic adaptations to SGLT2 inhibition and lay the groundwork for additional research, particularly relating to the paradoxical rise in EGP. Some questions are still left unanswered. Would a combination of SGLT2 inhibitors with agents such as incretins address the increase in EGP? Does the discrepancy between calories lost in the urine and total weight loss really indicate that SGLT2 inhibitors increase caloric intake? Clearly, additional metabolic studies are needed and should provide additional clarification.

\section{Acknowledgments}

W.T. Cefalu is supported in part by grant 1 U54 GM104940 from the National Institute of General Medical Sciences of the National Institutes of Health, which funds the Louisiana Clinical and Translational Science Center; by grant P50AT002776 from the National Center for Complementary and Alternative Medicine (NCCAM) and the Office of Dietary Supplements (ODS), which funds the Botanical Research Center; and from NIH grant P30DK074276, which funds the Nutrition Obesity Research Center.

Address correspondence to: William T. Cefalu, Douglas L. Manship, Sr. Professor Diabetes, Executive Director, Pennington Biomedical Research Center, 6400 Perkins Road, Baton Rouge, Louisiana 70808, USA. Phone: 225.763.2654; Fax: 225.763.3030; E-mail: cefaluwt@pbrc.edu.

1. Inzucchi SE, et al. Management of hyperglycemia in type 2 diabetes: a patient-centered approach. Position statement of the American Diabetes Association (ADA) and the European Association for the Study of Diabetes (EASD). Diabetes Care. 2012;35(6):1364-1379.

2. DeFronzo RA, Davidson JA, Del Prato S. The role of the kidneys in glucose homeostasis: a new path towards normalizing glycaemia. Diabetes Obes Metab. 2012;14(1):5-14

3. Monami M, Nardini C, Mannucci E. Efficacy and safety of sodium glucose co-transport-2 inhibitors in type 2 diabetes: a meta-analysis of randomized clinical trials [published online ahead of print December 5, 2013]. Diabetes Obes Metab. doi:10.1111/dom.12244.

4. Cefalu WT, et al. Efficacy and safety of canagliflozin versus glimepiride in patients with type 2 diabetes inadequately controlled with metformin (CANTATA-SU): 52 week results from a randomised, double-blind, phase 3 non-inferiority trial. Lancet. 2013;382(9896):941-950.

5. Goring SM, et al. Dapagliflozin compared with other oral anti-diabetes treatments when added to metformin monotherapy: a systematic review and network meta-analysis [published online ahead of print November 14, 2013]. Diabetes Obes Metab. doi:10.1111/dom.12239.

6. Merovci A, et al. Dapagliflozin improves muscle insulin sensitivity but enhances endogenous glucose production. J Clin Invest. 2014;124(2):509-514.

7. Ferrannini E, et al. Metabolic response to sodiumglucose cotransporter 2 inhibition in type 2 diabetic patients. J Clin Invest. 2014;124(2):499-508.

8. Rossetti L, Giaccari A, DeFronzo RA. Glucose toxicity. Diabetes Care. 1990;13(6):610-630.

9. DeFronzo RA, Ferrannini E, Simonson DC. Fasting hyperglycemia in non-insulin-dependent diabetes mellitus: contributions of excessive hepatic glucose production and impaired tissue glucose uptake. Metabolism. 1989;38(4):387-395.

10. Rossetti L, Smith D, Shulman GI, Papachristou D, DeFronzo RA. Correction of hyperglycemia with phlorizin normalizes tissue sensitivity to insulin in diabetic rats. J Clin Invest. 1987;79(5):1510-1515.

\title{
SMN-targeted therapeutics for spinal muscular atrophy: are we SMArt enough yet?
}

\author{
Kathryn J. Swoboda
}

Department of Neurology, University of Utah School of Medicine, Salt Lake City, Utah, USA.

\begin{abstract}
Spinal muscular atrophy (SMA) remains one of the most common and lethal autosomal recessive diseases. Homozygous deletion of survival of motor neuron 1 (SMN1) and resulting SMN protein deficiency manifests predominantly with motor neuron degeneration; however, a wealth of emerging data supports a broader influence of SMN deficiency in disease pathogenesis. In this issue of the JCI, Kariya and colleagues demonstrate the relatively selective impact of SMN depletion on the distal motor unit using a series of SMN2-expressing transgenic mice in which constitutive SMN knockdown follows variable periods of normal development. Their observations provide further insights regarding the temporal requirements for SMN in mice, renewing speculation about when and where repletion of SMN is necessary for optimal outcomes in SMA patients.
\end{abstract}

\section{SMN-targeted therapeutics: the hope and the hype}

Spinal muscular atrophy (SMA) is an autosomal recessive disorder characterized by motor neuron loss in the spinal

Conflict of interest: Kathryn J. Swoboda has clinical trial contracts with ISIS Pharmaceuticals and Roche Pharmaceuticals for clinical studies or trials in SMA patients.

Citation for this article: J Clin Invest. 2014; 124(2):487-490. doi:10.1172/JCI74142. cord and brainstem. Progressive muscular weakness and atrophy typically emerge in infancy or early childhood after a variable period of normal development. Deficiency in survival motoneuron ( $\mathrm{SMN}$ ) is associated with SMA. In humans and bonobos, a unique inverted duplication on chromosome 5 encompasses SMN1 and its nearly identical homolog, SMN2; however, lower vertebrates, including mice, lack SMN2. Deletion and/or mutation of SMN1 is dis- ease causing, and a translationally silent nucleotide substitution in SMN2 results in a truncated, less stable protein. Historically, low SMN was thought to selectively target motor neurons; however, emerging data from SMA mouse models indicate that low levels of SMN affect many tissues, including components of the neuromuscular circuitry, and skeletal muscle (1). In addition to obvious defects in neuromuscular junction (NMJ) maturation, decreased SMN results in early abnormalities in synaptic input from muscle fibers to motor neurons within the spinal cord $(2,3)$. Both SMA mouse models and severely affected human infants exhibit abnormalities in myofiber maturation, muscle size, and muscle function (4-6). Furthermore, there is evidence that $\mathrm{SMN}$-intrinsic defects in Schwann cells promote SMA pathogenesis, and peripheral nerve myelination is abnormal in severely affected infants (7). In humans lacking SMN production from SMN1, SMN2 phenotypically modifies 
A

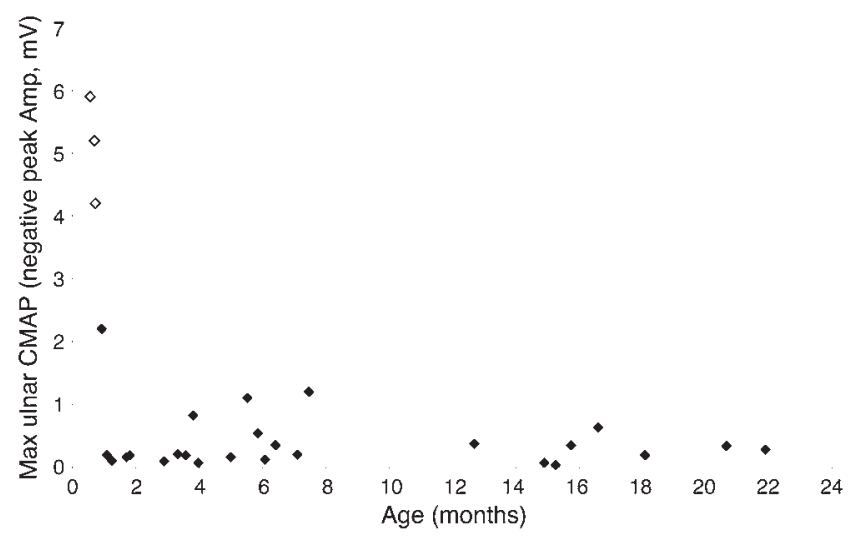

C

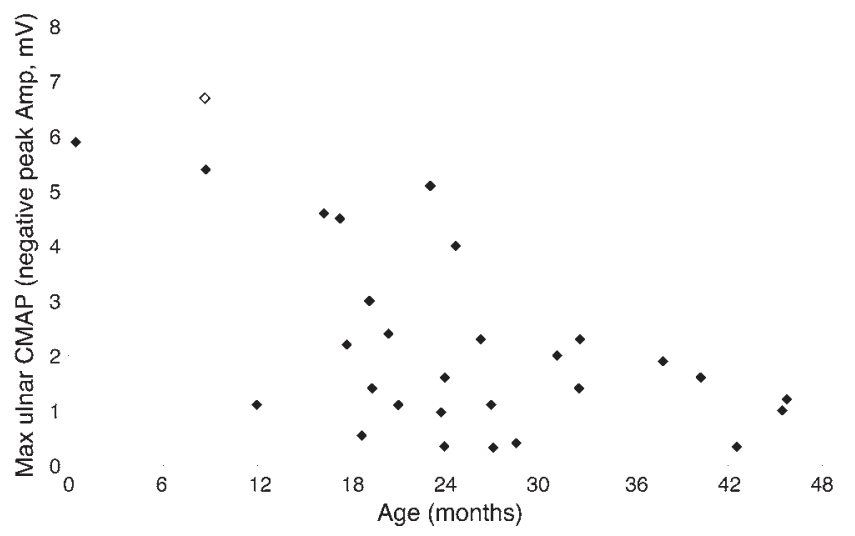

B

300

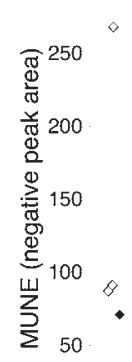

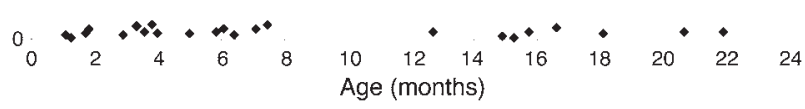

D

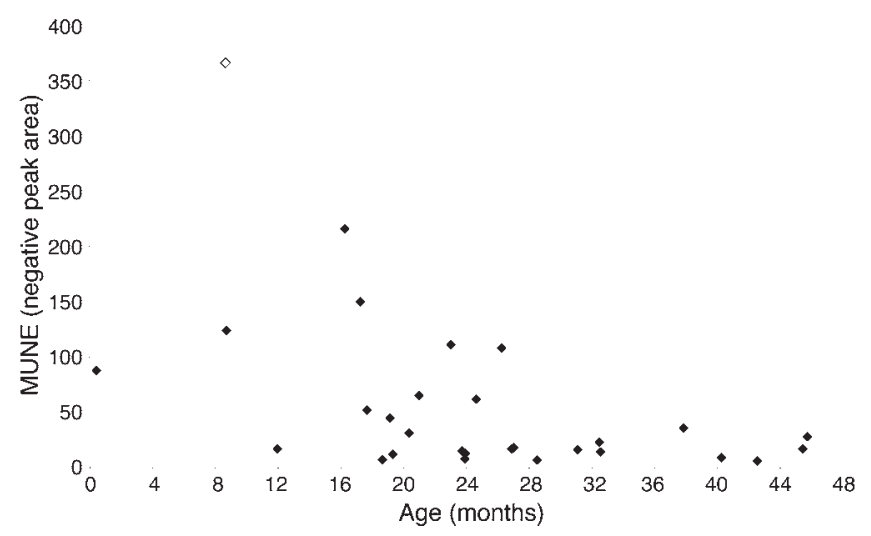

Figure 1

Denervation status in relation to age in SMA type I and SMA type II. (A) Maximum ulnar CMAP amplitude and (B) MUNE values versus age in SMA type I infants, birth to 24 months. (C) Maximum ulnar CMAP amplitude and (D) MUNE values versus age in SMA type II children from birth to 48 months of age. For all panels, white diamonds indicate presymptomatic infants (identified via genetic testing due to an affected sibling). Black diamonds indicate already symptomatic infants. Adapted with permission from Annals of Neurology (Figures 2 and 3; ref. 20).

SMA severity, with SMN2 dosage inversely correlating with disease severity. In mice, Smn deletion results in embryonic lethality; however, introduction of multiple copies of SMN2 into $\mathrm{Smn}^{-1-}$ mice ameliorates disease phenotypes (8). Because SMN2 is universally present in SMA patients, it is a compelling therapeutic target for SMA-associated neurodegenerative disease. SMN-targeted therapies are already being evaluated in clinical trials, increasing the urgency for understanding when and where SMN is required and the best delivery mode for effective SMN-mediated prevention, stabilization, or reversal of motor neuron degeneration. Accumulating evidence suggests that abnormalities of the motor neuron cell body and its central synapses, the axon, the NMJ, and skeletal muscle contribute synergistically to SMA pathogenesis and that $\mathrm{SMN}$ repletion within both central and peripheral nervous systems may be required for beneficial therapeutic outcomes $(9,10)$.

\section{The ubiquitous SMN protein: here, there, and everywhere}

All cells require SMN for biogenesis of snRNP particles and pre-mRNA splicing. If there is a universal requirement for SMN, why are motor neurons selectively affected by its loss, and where does SMA first manifest? Does SMN dysfunction begin at the level of the motor neuron cell body, the axon, or the NMJ $(11,12)$, and what effect does reduced SMN have on other components of the neuromuscular circuitry (13)? In zebrafish, SMN is required in motor neurons for normal growth and connectivity (14); however, SMA mice, which die within two to three weeks postnatally, display normal motor neuron connectivity at birth and only minimal abnormalities in distal NMJs during prenatal and neonatal development $(15,16)$. Several groups have also demonstrated that early postnatal induction of SMN is critical for prolonged survival in transgenic SMA mouse models $(17,18)$. In order to address the precise temporal requirements for SMN in postnatal development, Kariya and colleagues developed mouse models in which SMN knockdown could be temporally induced (19). By generating a series of transgenic mice with variable SMN2 copy number and inducible SMN knockdown, Kariya et al. were able to evaluate the impact of SMN depletion both constitutively and at multiple time points in postnatal development. Kariya and colleagues determined that prior to P15, motor unit development is vulnerable to abrupt SMN knockdown in the presence of two SMN2 copies; however, mature (after P50) mice with two copies of SMN2 were not markedly affected by SMN knockdown (19). Interestingly, as noted by 
Kariya et al., the emergence of this relative resistance to SMN depletion at $\mathrm{P} 15$ in the mouse correlates morphologically with maturation of the distal NMJs (19).

\section{Back to reality}

Unfortunately, the most frequently inherited genotype in humans, homozygous SMN1 deletion with two SMN2 copies, is associated with the severe infantile phenotype (SMA type I), which accounts for about half of all newly diagnosed SMA patients each year. There are limited data regarding reversibility of SMA phenotypes in these often severely weak infants, who are currently diagnosed at a mean age of approximately 4.9 months (Families of Spinal Muscular Atrophy [FSMA] registry data from 2012; Jill Jarecki, personal communication). Compared with neonatal SMA mice, infants with SMA type I have more precipitous and severe denervation, and autopsies of infants that have died soon after their diagnosis demonstrate severe motor neuron loss within the spinal cord (C.J. Sumner and K.J. Swoboda, unpublished data).

While the critical SMN-sensitive period identified by Kariya et al. encompasses the period of maturation of the distal motor unit, it also spans a period of tremendous growth and developmental change (19). The translation of observations in mice to human disease remains a major challenge for scientists and clinicians trying to reach conclusions about the relevance of any preclinical observations, even those obtained under the most rigorously controlled experimental conditions. Despite these challenges, interesting parallels between mouse SMA models and human infants with SMA should be taken under consideration. The critical period in mice for distal NMJ maturation corresponds to the onset of weaning, as animals transition from suckling to mastication. Mice demonstrate tremendous growth during the first few weeks after birth, increasing their body weight sevento eight-fold. Such rapid growth requires the motor unit to accommodate growth of the primary peripheral target, skeletal muscle, and to meet the needs of the motor neuron to manage axonal extension and distal NMJ remodeling. Like their murine counterparts, presymptomatic infants with SMA appear to have normal distal motor connectivity at birth.

Previously, we demonstrated the relationship among SMA type, SMN2 copy number, motor function, and denervation status in 89 SMA children enrolled in a natural history study at the University of Utah (20). Denervation status in a distally innervated hand muscle was studied using two electrophysiologic techniques: the maximum ulnar compound muscle action potential (CMAP) and a technique for motor unit number estimation (MUNE). Evaluation of CMAP and MUNE data from SMA type I infants presenting from birth to two years of age revealed a drastic denervation in these infants by two months of age (Figure 1, A and B, and ref. 20). Furthermore, we documented precipitous denervation within weeks to months after birth in a small subset of presymptomatic type I infants within this cohort (see Figure 4 of ref. 20). Since this publication, we have extended these observations to more than 80 type I and 150 type II SMA infants and children (K.J. Swoboda, unpublished data). SMA type I infants demonstrate an almost logarithmic decline in CMAP and MUNE values with age, reaching a nadir by four to six months, and associated with the development of generalized hypotonia and weakness. In contrast with infants with type I SMA, we observed that denervation in infants and children with SMA type II is much less acute and more variable, with the most active denervation occurring between six and 36 months of age (ref. 20 and Figure 1, C and D). Together, these observations support that human infants with a given SMN genotype demonstrate a vulnerability to disease progression during a relatively specific and critical period of development, similar to the observations in a murine model of SMA by Kariya and colleagues. This window of disease progression in SMA infants occurs during a period of rapid growth, peripheral neuromuscular development, and central nervous system development, as well as peripheral and central nervous system myelination. Additionally, in type I infants, this time period correlates with the transition from central pattern-generated reflex suckling to a more modulated pattern of nutritional intake, as infants advance from a liquid diet to a diet including solids.

\section{What does this mean for clinical trials?}

For years, clinicians have been stymied by the distinctive bimodal pattern of SMAassociated progression of neurodegeneration, which is perhaps most aptly characterized by Tom Crawford as "a slowing of the rate of degeneration with the passage of time" (21). Bimodal disease progression is uncommon in neurodegenerative disorders and makes an accurate prognosis following SMA diagnosis challenging. There is some overlap in the age of SMA onset in infants the severe form (type I) and those with the intermediate form (type II). While most SMA infants who present with weakness within the first year but are able sit unsupported will manifest an intermediate phenotype (type II), some will go on to ambulate independently (type IIIa; ref. 22).

Patterns of disease progression that vary by age and SMA subtype make clinical trial design challenging. Most children with SMA type II or III also present with an acute phase of denervation and clinical disease progression, albeit somewhat less obviously than those with SMA type I. Because the initial presentation of symptoms is followed by a period of stabilization of motor function that can last for months or even years, it is a challenge to accurately diagnose SMA subtype and provide meaningful prognosis. Additionally, SMA subtype, SMN genotype, modifying genetic factors, and environmental factors such as nutritional state or superimposed illness can all contribute to phenotypic heterogeneity. While an SMN2 copy number of two or less in the setting of symptom onset in early infancy remains a strong predictive determinant for developing SMA type I, identification of intrinsic and extrinsic factors that influence disease outcome has increased the urgency for better predictive and prognostic SMA biomarkers. Development of additional SMA biomarkers would allow for better characterization of SMA patient cohorts for early clinical trial enrollment, allowing for better assessment of SMA therapies.

\section{Conclusions and future directions}

Building upon previous studies, Kariya and colleagues demonstrate that SMN requirements are higher during early development through the completion of maturation of the distal NMJ and that acute depletion in adulthood results in minimal motor neuron dysfunction in mice with at least two SMN2 copies. Finally, the study by Kariya et al. demonstrates that SMN reduction after mice have reached maturity impairs reinnervation following peripheral nerve injury (19).

In their discussion, Kariya and colleagues boldly translate observations from SMA mouse models to human subjects, speculating that once effective SMN-targeted therapeutics are identified, they may be tapered 
at a future time point. Given the major differences in time to maturation and life span between mice and humans, translating SMA-sensitive periods from mouse models to humans remains a major challenge. If SMA mouse models prove to accurately represent what might be expected in human clinical trials, then early or even presymptomatic intervention may be required to meet FDA efficacy standards. Because SMA type I infants present with generalized muscle weakness within six months of birth, newborn screening may be the only realistic means for preserving motor function in these patients. Patients with milder forms of SMA account for a substantial proportion of surviving patients and demonstrate a slower rate of denervation, providing a longer window of opportunity for therapeutic intervention. Due to the high stakes of therapeutics development, setting unrealistic efficacy goals could doom promising therapies that could benefit SMA patients with milder disease forms. For therapeutic trials targeting the most fragile patients, symptomatic type I infants, we must remain conscious that in some cases, a modest benefit simply is not enough.

While caution is warranted, the future is bright for SMN-targeted therapeutics, and the opportunity to dramatically improve or even cure SMA remains tantalizingly within reach. Given the continued emergence of data in both animal models and humans that SMN has the greatest impact on motor neurons during a critical developmental window, early intervention has never been more appealing. Heeding the lessons provided to us by the increasing wealth of data from a variety of experiments across diverse animal models will undoubtedly permit us to realize the greatest impact for our human patients.

\section{Acknowledgments}

The author has received grant funding from the National Institutes of Child Health and
Human Development, the National Institutes of Neurologic Disease and Stroke, FightSMA, Families of SMA, and the Muscular Dystrophy Association in support of SMA-related research. This work was supported in part by NIH grants NICHD R01-HD054599 and R01-HD69045 and by the National Center for Advancing Translations Sciences (award no. 1ULTR001067). This content is solely the responsibility of the author and does not necessarily represent the official views of the NIH.

Address correspondence to: Kathryn J. Swoboda, Pediatric Motor Disorders Research Program, Department of Neurology, 30 N. 1900 E. SOM3R149, Salt Lake City, Utah 84132, USA. Phone: 801.585.9717; Fax: 801.587.9346; E-mail: Swoboda@genetics.utah.edu.

1. Sleigh JN, Gillingwater TH, Talbot K. The contribution of mouse models to understanding the pathogenesis of spinal muscular atrophy. Dis Model Mech. 2011;4(4):457-467.

2. Ling KK, Lin MY, Zingg B, Feng Z, Ko CP. Synaptic defects in the spinal and neuromuscular circuitry in a mouse model of spinal muscular atrophy. PLoS One. 2010;5(11):e15457.

3. Mentis GZ, et al. Early functional impairment of sensory-motor connectivity in a mouse model of spinal muscular atrophy. Neuron. 2011;69(3):453-467.

4. Nadeau A, D’Anjou G, Debray G, Robitaille Y, Simard LR, Vanasse M. A newborn with spinal muscular atrophy type 0 presenting with a clinicopathological picture suggestive of myotubular myopathy. J Child Neurol. 2007;22(11):1301-1304.

5. Lee YI, Mikesh M, Smith I, Rimer M, Thompson W. Muscles in a mouse model of spinal muscular atrophy show profound defects in neuromuscular development even in the absence of failure in neuromuscular transmission or loss of motor neurons. Dev Biol. 2011;356(2):432-444.

6. Boyer JG, Murray LM, Scott K, De Repentigny Y, Renaud JM, Kothary R. Early onset muscle weakness and disruption of muscle proteins in mouse models of spinal muscular atrophy. Skelet Muscle. 2013;3(1):24.

7. Hunter G, Aghamaleky Sarvestany A, Roche SL, Symes RC, Gillingwater TH. SMN-dependent intrinsic defects in Schwann cells in mouse models of spinal muscular atrophy [published online ahead of print December 16, 2013]. Hum Mol Genet. doi: $10.1093 / \mathrm{hmg} / \mathrm{ddt} 612$.

8. Monani UR, et al. The human centromeric survival motor neuron gene (SMN2) rescues embryonic lethality in $\operatorname{Smn}(-/-)$ mice and results in a mouse with spinal muscular atrophy. Hum Mol Genet. 2000; 9(3):333-339

9. Hua Y, et al. Peripheral SMN restoration is essential for long-term rescue of a severe spinal muscular atrophy mouse model. Nature. 2011; 478(7367):123-126.

10. Martinez TL, et al. Survival motor neuron protein in motor neurons determines synaptic integrity in spinal muscular atrophy. J Neurosci. 2012; 32(25):8703-8715.

11. d'Errico P, et al. Selective vulnerability of spinal and cortical motor neuron subpopulations in delta7 SMA mice. PLoS One. 2013;8(12):e82654.

12. Fallini $C$, et al. Dynamics of survival of motor neuron $(\mathrm{SMN})$ protein interaction with the mRNA-binding protein IMP1 facilitates its trafficking into motor neuron axons [published online ahead of print July 29, 2013]. Dev Neurobiol. doi:10.1002/dneu.22111.

13. Gogliotti RG, Quinlan KA, Barlow CB, Heier CR, Heckman CJ, Didonato CJ. Motor neuron rescue in spinal muscular atrophy mice demonstrates that sensory-motor defects are a consequence, not a cause, of motor neuron dysfunction. J Neurosci. 2012; 32(11):3818-3829.

14. Hao le T, Duy PQ, Jontes JD, Wolman M, Granato $\mathrm{M}$, Beattie CE. Temporal requirement for SMN in motoneuron development. Hum Mol Genet. 2013;22(13):2612-2625.

15. McGovern VL, Gavrilina TO, Beattie CE, Burghes AH. Embryonic motor axon development in the severe SMA mouse. Hum Mol Genet. 2008; 17(18):2900-2909.

16. Murray LM, Lee S, Bäumer D, Parson SH, Talbot K, Gillingwater TH. Pre-symptomatic development of lower motor neuron connectivity in a mouse model of severe spinal muscular atrophy. Hum Mol Genet. 2010; 19(3):420-433.

17. Hammond SM, Gogliotti RG, Rao V, Beauvais A, Kothary R, DiDonato CJ. Mouse survival motor neuron alleles that mimic SMN2 splicing and are inducible rescue embryonic lethality early in development but not late. PLoS One. 2010;5(12):e15887.

18. Le TT, et al. Temporal requirement for high SMN expression in SMA mice. Hum Mol Genet. 2011; 20(18):3578-3591.

19. Kariya S, et al. Requirement of enhanced Survival Motoneuron protein imposed during neuromuscular junction maturation. J Clin Invest. 2014; 124(2):785-800.

20. Swoboda KJ, et al. Natural history of denervation in SMA: Relation to age, SMN2 copy number, and function. Ann Neurol. 2005;57(5):704-712.

21. Crawford TO. Concerns about the design of clinical trials for spinal muscular atrophy. Neuromuscul Disord. 2004;14(8-9):456-460.

22. Zerres K, Rudnik-Schöneborn S. Natural history in proximal spinal muscular atrophy. Clinical analysis of 445 patients and suggestions for a modification of existing classifications. Arch Neurol. 1995; 52(5):518-523. 